\title{
Suppressed epithelial-mesenchymal transition and cancer stem cell properties mediate the anti-cancer effects of ethyl pyruvate via regulation of the AKT/nuclear factor- $\kappa B$ pathway in prostate cancer cells
}

\author{
BIN HUANG $^{1 *}$, DAO-JUN LV ${ }^{1 *}$, CHONG WANG $^{1}$, FANG-PENG SHU $^{1}$, ZHI-CHENG GONG $^{1}$, \\ TAO XIE ${ }^{1}$, YU-ZHONG YU ${ }^{1}$, XIAN-LU SONG ${ }^{2}$, JIA-JIA XIE ${ }^{3}$, SEN LI $^{3}$, \\ YA-MENG LIU ${ }^{3}$, HUAN QI ${ }^{1,3}$ and SHAN-CHAO ZHAO ${ }^{1,3}$

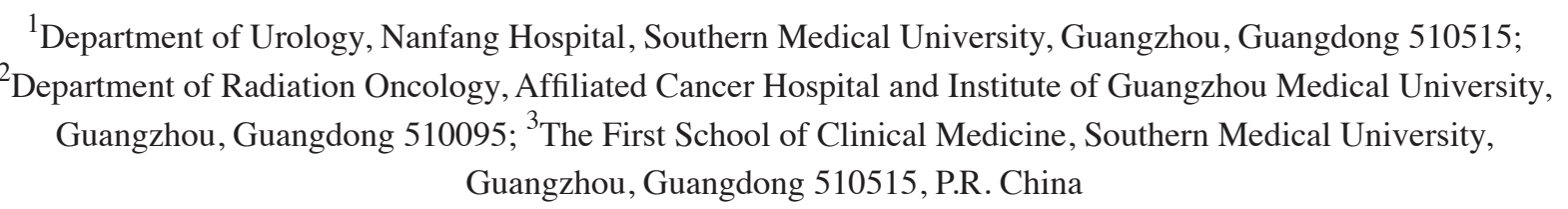

Received January 26, 2018; Accepted May 31, 2018

DOI: $10.3892 / \mathrm{ol} .2018 .8958$

\begin{abstract}
Castration-resistant prostate cancer (CRPC) is a leading cause of mortality among cases of prostate cancer (PCa). Current treatment options for CRPC are limited. Ethyl pyruvate (EP), a lipophilic derivative of pyruvic acid, has been reported to have antitumor activities. In the present study, the efficacy of EP against PCa was investigated using two human PCa cell lines and a mouse xenograft tumor model. PC3 and CWR22RV1 cells were treated with EP, and cytotoxicity was evaluated via Cell Counting Kit-8 and colony formation assays, while cell cycle distribution was assessed by flow cytometry. Changes in cell migration and invasion caused by EP treatment were also evaluated with Transwell and wound healing assays, and changes in the expression of intracellular signaling pathway components were detected by western blotting. EP treatment reduced cell viability, induced G1 arrest, and activated the intrinsic
\end{abstract}

Correspondence to: Professor Shan-Chao Zhao or Dr Huan Qi, Department of Urology, Nanfang Hospital, Southern Medical University, 1838 GuangZhou Avenue, Guangzhou, Guangdong 510515, P.R. China

E-mail: zhaoshanchaosmu@sina.cn

E-mail:2668241842@qq.com

*Contributed equally

Abbreviations: ADT, androgen-deprivation therapy; PCA, prostate cancer; CRPC, castration-resistant prostate cancer; EMT, epithelial-mesenchymal transition; EP, ethyl pyruvate; TNF, tumor necrosis factor; CDK, cyclin-dependent kinase; PARP, poly(ADP-ribose) polymerase

Key words: prostate cancer, ethyl pyruvate, epithelial-mesenchymal transition, cancer stem cells, AKT/nuclear factor- $x \mathrm{~B}$ apoptosis pathway. Additionally, the in vivo experiments revealed that EP administration markedly inhibited tumor growth. EP also reversed epithelial-mesenchymal transition and suppressed cancer stem cell properties in part through negative regulation of $\mathrm{AKT} /$ nuclear factor $-\kappa \mathrm{B}$ signaling. These results indicate that $\mathrm{EP}$ has anticancer activity in vitro and in vivo, and is therefore a promising therapeutic agent for the treatment of PCa.

\section{Introduction}

Prostate cancer $(\mathrm{PCa})$ is the most commonly diagnosed malignancy and causes over 30,000 fatalities each year in men in the USA (1). Advanced PCa is routinely treated by androgen deprivation therapy (ADT); however, it can eventually progress to castration-resistant prostate cancer (CRPC) (2). Recently developed ADT drugs including enzalutamide and abiraterone acetate can improve survival (3), but the overall prognosis of patients with CRPC remains poor (4). Therefore, more effective and less toxic agents are required for CRPC therapy.

Epithelial-to-mesenchymal transition (EMT) is a tightly regulated biological process in which epithelial cells acquire mesenchymal characteristics and upregulate the expression of mesenchymal markers, and is associated with malignant progression (5). Cancer cells that have undergone EMT via activation of the nuclear factor $(\mathrm{NF})-\kappa \mathrm{B}$, Wnt and Hedgehog signaling pathways acquire stem cell-like traits, including the capacity for self-renewal and differentiation (6,7). Cancer stem cells (CSCs) form spheres in non-adherent cultures and express Nanog, Octamer-binding protein 4 (OCT4) and cluster of differentiation 44 (CD44) (8). An EMT-like state and CSC features are associated with metastasis and resistance to ADT $(9,10)$, and are thus ideal therapeutic targets for CRPC treatment.

Ethyl pyruvate (EP) is a stable aliphatic ester derived from pyruvic acid that has been demonstrated to suppress 
inflammation, mitigate redox-mediated cellular damage, and exert immunoregulatory and neuroprotective effects $(11,12)$. It also has anti-tumor effects in many types of cancer, including gallbladder cancer, lung adenocarcinoma and hepatic tumors, among others (13-18). However, the molecular basis for these effects is not well understood and, to the best of our knowledge, they have not been investigated in the context of PCa.

To address these issues, in the current study the antitumor effects of EP were investigated in PCa cells lines and a mouse xenograft model. The results indicated that EP may block tumor growth, migration and EMT, and stimulate apoptosis, and may thus be a promising treatment modality for PCa.

\section{Materials and methods}

Cell lines. The human PCa cell lines PC3 and CWR22RV1 were purchased from the Cell Bank of the Chinese Academy of Sciences (Shanghai, China). The cells were cultured as previously described (19).

Reagents and materials. The following antibodies were used in the present study: Rabbit polyclonal anti-E-cadherin (cat. no. 0874-1-AP, 1:500), - $\beta$-actin (cat. no. 60008-1-Ig), -tubulin (cat. no. 11224-1-AP), -vimentin (cat. no. 10366-1-AP) and -p21 (cat. no. 10355-1-AP) (1:1,000; all from ProteinTech, Rosemont, IL, USA); rabbit anti-cyclin D1 (cat. no. 2978T), -cyclin-dependent kinase (CDK)4 (cat. no. 12790T), -poly (ADP-ribose) polymerase (PARP) (cat. no. 9532T), -AKT (cat. no. 4691T) and -p65 (cat. no. 8242S) (all 1:1,000); rabbit anti-caspase 3 (cat. no. 9662S), -cleaved caspase-3 (9664T), -cleaved PARP (cat. no. 5625T), -phosphorylated (p)-AKT (SER473) (cat. no. 4060T) and -p-p65 (cat. no. 3033T) (all 1:500; all from Cell Signaling Technology, Inc., Danvers, MA, USA); and mouse anti-histone-3 (cat. no. ab1791, 1:1,000; Abcam, Cambridge, UK). EP and human recombinant tumor necrosis factor (TNF)- $\alpha$ were obtained from Shanghai Macklin Biochemical Co., Ltd., (Shanghai, China) and Sigma-Aldrich; Merck KGaA, (Darmstadt, Germany), respectively.

Cell viability test. PC 3 and CWR22RV1 cells (5x10\%3ell) were seeded in 96-well plates and grown until they had adhered to the plate. They were then treated with $5,10,15$ or $20 \mathrm{mM}$ EP for 24,48 or $72 \mathrm{~h}$. Cell viability was evaluated with a Cell Counting Kit (CCK)-8 (Dojindo Molecular Technologies, Inc., Kumamoto, Japan) according to the manufacturer's instructions. Briefly, cells were incubated for $2 \mathrm{~h}$ in the CCK-8 solution (10 $\mu \mathrm{l} /$ per well) and the absorbance was read at $490 \mathrm{~nm}$.

Colony formation assay. The effect of EP on PCa cell growth was evaluated with a colony formation assay. PC3 cells (500/well) and CWR22RV1 cells (800/well) were seeded in a 6 -well plate (Corning Inc., Corning, NY, USA) and cultured for 2 weeks. The cells were fixed with methanol and stained with Giemsa. Colonies containing at least 50 cells were counted.

Wound healing assay. PCa cells (1.5×10\%/per well) were seeded in a 6-well plate and incubated overnight until they reached $75-90 \%$ confluence. The cell monolayer was scratched with a $10-\mu 1$ plastic pipette tip. After washing twice with PBS to remove cell debris, Roswell Park Memorial Institute
1640 medium containing $1 \%$ fetal bovine serum (FBS) and 10 or $15 \mathrm{mM}$ EP or PBS was added. After $24 \mathrm{~h}$, the cells were photographed and the migrated distance was measured with ImageJ software (National Institutes of Health, Bethesda, MD, USA).

Transwell invasion assay. Cells were resuspended in serum-free 1640 medium containing 10 or $15 \mathrm{mM}$ EP or PBS. Cells $\left(5 \times 10^{4} / 200 \mu \mathrm{l}\right)$ were added to the upper compartment of a Transwell chamber containing Matrigel. The lower compartment was filled with $600 \mu \mathrm{l}$ medium containing $20 \%$ FBS. After incubation for $12 \mathrm{~h}$, a cotton swab was used to wipe the top chamber and remove the non-migratory cells, and migrated cells attached to the bottom side of the chamber were fixed with methanol and stained with Giemsa.

Cell cycle analysis. A Cell Cycle Staining kit (Hangzhou MultiSciences (Lianke) Biotech, Co., Ltd., Shanghai, China) was used to evaluate cell cycle distribution according to the manufacturer's instructions. Briefly, cells were incubated with $15 \mathrm{mM}$ EP for $48 \mathrm{~h}$, dissociated by trypsinization $(0.25 \%$ trypin $+0.02 \%$ EDTA), and concentrated by centrifugation (2,000 rpm, $5 \mathrm{~min})$. Following washing with PBS, the cells were resuspended in $1 \mathrm{ml}$ DNA staining solution and $10 \mu \mathrm{l}$ permeabilization solution, then incubated at room temperature in the dark for $30 \mathrm{~min}$. DNA content was determined by flow cytometry on a FACSCalibur instrument (BD Biosciences, Franklin Lakes, NJ, USA).

Sphere formation assay. The stemness potential of EP-treated PCa cells was evaluated with a sphere formation assay as previously described (20).

Nuclear extract preparation. PC3 and CWR22RV1 cells were pretreated with $15 \mathrm{mM}$ EP for $6 \mathrm{~h}$ and then stimulated with $10 \mathrm{ng} / \mathrm{ml} \mathrm{TNF}-\alpha$ for $2 \mathrm{~h}$. Total cell lysate was separated into cytoplasmic and nuclear components using a Nuclear-Cytosol Extraction kit (Nanjing KeyGen Biotech Co., Ltd., Nanjing, China).

Western blot analysis. The total cell lysate of PCa cells treated with $15 \mathrm{mM}$ EP was analyzed by sodium dodecyl sulfate polyacrylamide gel electrophoresis (SDS-PAGE) on a 4-12\% gel prepared with an SDS-PAGE Gel Preparation kit (Nanjing KeyGen Biotech Co., Ltd.), with $30 \mu$ g protein loaded in each well. The proteins were transferred to a $0.45-\mu \mathrm{m}$ polyvinylidene difluoride membrane that was probed with the indicated antibodies.

Evaluation of the antitumor potential of EP in vivo. Male BALB/C nu/nu mice (6 weeks old) were purchased from the Laboratory Animal Center of Southern Medical University. A total of $5 \times 10^{6}$ PC3 cells were resuspended in PBS and injected into the right upper limb of each mouse. When subcutaneous tumors had formed after approximately 7 days, 10 mice were randomly allocated into control or treatment groups and intraperitoneally injected once daily for 2 weeks with $50 \mathrm{mg} / \mathrm{kg}$ EP or saline, respectively. Procedures involving animals were approved by the Institutional Animal Care and Use Committee of Southern Medical University. 

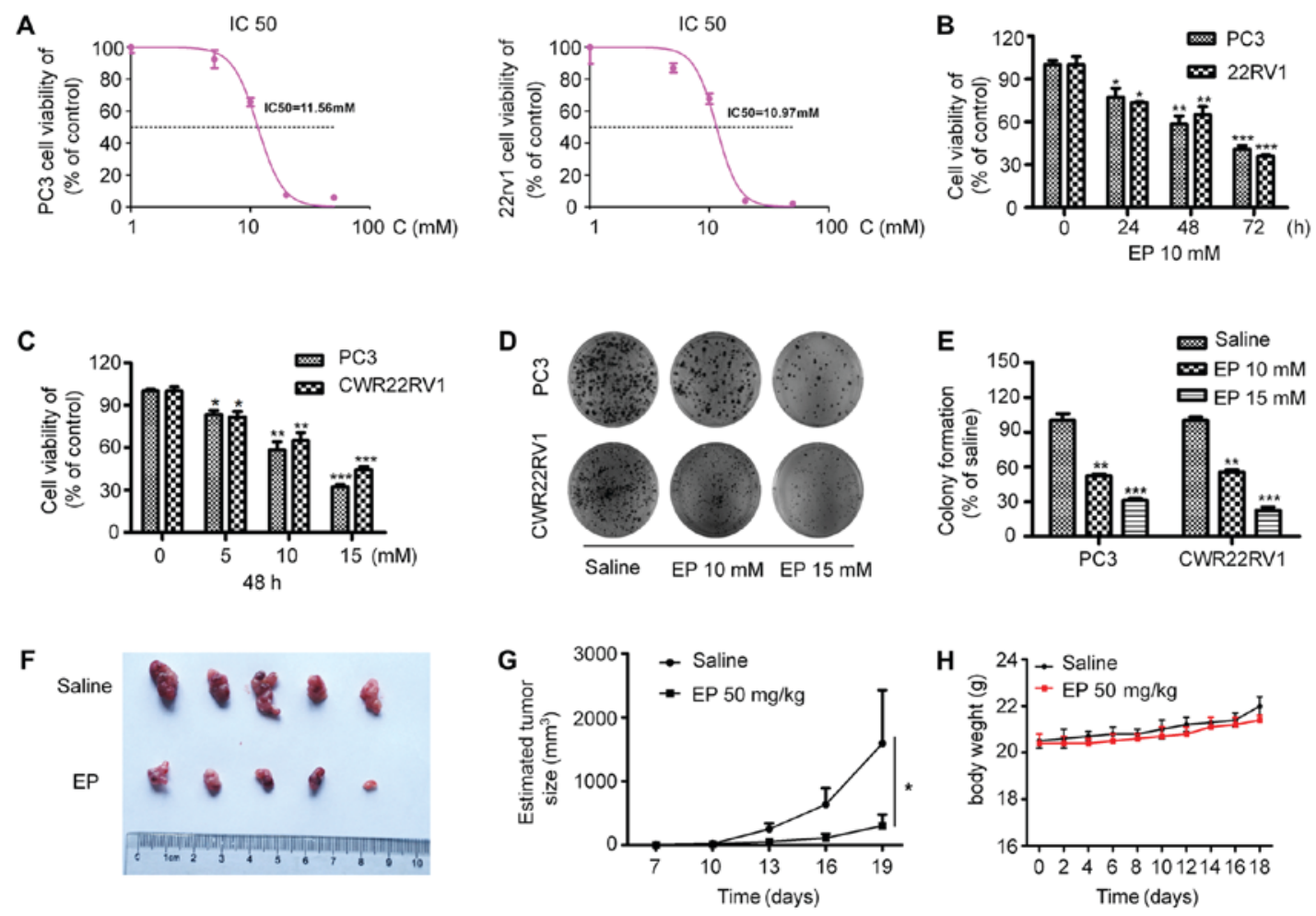

Figure 1. Effect of EP on PCa cell viability, cell colony formation and tumourigenesis. (A) EP show cytotoxic potential against the PC3 and 22Rv1 with IC50 values of $11.56 \mathrm{mM}$ and $10.93 \mathrm{mM}$, respectively. (B) The kinetics of cell viability in PC3 and CWR22RV1 cells treated with EP. Cells were treated with EP (10 mmol/1) at the indicated time. (C) The dosage effect of EP on the cell viability in PC3 and CWR22RV1 cells. (D) The tumor cell colony formation was analyzed, and (E) the colony formation rate was calculated. The data represented means $\pm \mathrm{SEM}\left({ }^{*} \mathrm{P}<0.05,{ }^{* * *} \mathrm{P}<0.01,{ }^{* * * *} \mathrm{P}<0.001 \mathrm{n}=3\right)$ as the percentage of viable cells normalized to percentage of viable cells in saline-treated (control) cells. (F) Gross observation of xenograft tumour size. (G) Plot of tumour volume over time. $(\mathrm{H})$ Body weight of tumor-bearing mice. Significant differences are between EP treatment groups and Saline vehicle control groups. EP, ethyl pyruvate; $\mathrm{PCa}$, prostate cancer; means $\pm \mathrm{SEM}$, mean \pm standard error of mean.

Terminal deoxynucleotidyl transferase dUTP nick end labeling (TUNEL) assays. Formalin-fixed paraffin-embedded Tumor tissues from the saline-treated group and the EP-treated groups were analyzed with TUNEL assays. At first, Tissue slice were deparaffinized with xylene and dehydrated with ethanol. And then, the slices were rinsed twice with PBS and treated with proteinase $\mathrm{K}(15 \mu \mathrm{g} / \mathrm{ml}$ in $10 \mathrm{mM}$ Tris/ $\mathrm{HCl}$, $\mathrm{pH} 7.4-8.0$ ) for $15 \mathrm{~min}-20 \mathrm{~min}$ at $37^{\circ} \mathrm{C}$. Endogenous peroxidases were blocked with $3 \%$ hydrogen peroxide in methanol at room temperature for $10 \mathrm{~min}$. The tissue sections were then analyzed with an in situ Cell Death Detection kit, POD (Nanjing KeyGen Biotech Co., Ltd.,), in accordance with the manufacturer's instructions. The reaction was visualized with fluorescence microscopy.

Statistical analysis. Data were statistically calculated using two-tailed Student's t-tests (two groups) or one-way ANOVA followed by the least significant difference post-hoc test (for more than two groups). $\mathrm{P}<0.05$ was considered to indicate a statistically significant difference. Value are presented as the means \pm standard deviation (SD) by GraphPad Prism software (GraphPad Software, Inc., La Jolla, CA, USA).

\section{Results}

EP inhibits the proliferation of human PCa cells in vitro and abolishes the tumor-forming capacity of PCa cells in vivo. To investigate the effects of EP on the proliferation of $\mathrm{PCa}$ cells, PC3 and CWR22RV1 cells were treated with different concentrations of EP for various times. EP show cytotoxic potential against the PC3 and 22Rv1 with IC50 values of 11.56 and $10.93 \mathrm{mM}$, respectively (Fig. 1A). EP treatment suppressed the proliferation of both cell lines in a concentration- and time-dependent manner ( $\mathrm{P}<0.05$; Fig. $1 \mathrm{~B}$ and $\mathrm{C})$. Furthermore, EP markedly inhibited the colony formation capacities of the cells $(\mathrm{P}<0.05$; Fig. $1 \mathrm{D}$ and $\mathrm{E})$. It was next investigated whether EP can suppress tumor growth in vivo. PC 3 cells were injected into the upper limbs of male nude mice, with EP administered after tumors had formed. Treatment with $50 \mathrm{mg} / \mathrm{kg}$ EP for 2 weeks significantly reduced tumor growth relative to vehicle-treated controls, resulting in smaller tumors $(\mathrm{P}<0.05$; Fig. $1 \mathrm{~F}$ and $\mathrm{G})$ by the end of the experimental period (average volume: $303.4 \pm 176.6$ vs. $1,594.6 \pm 834.7 \mathrm{~mm}^{3}$ ). Correspondingly, the EP treatment was well tolerated and did not result in any significant in vivo toxicity (Fig. 1H).

EP induces G1 arrest and apoptosis. To investigate whether the anti-proliferative effects of EP on PCa cells were associated with changes in cell cycle progression, PC3 and CWR22RV1 cells were treated with $15 \mathrm{mM}$ EP for $48 \mathrm{~h}$ and cell cycle distribution was analyzed by flow cytometry. The G1 fraction was markedly increased following treatment with EP relative to the negative control group (Fig. 2A). Similar results were obtained in CWR22RV1 cells (Fig. 2A). A western blot analysis revealed that the treatment with EP decreased the expression of cyclin D1 and CDK4 and increased that of p21 (Fig. 2B). 
A
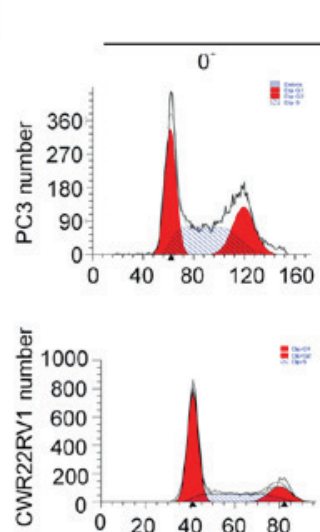

B

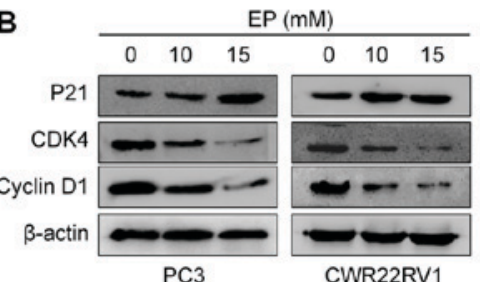

$\mathrm{EP}(\mathrm{mM})$
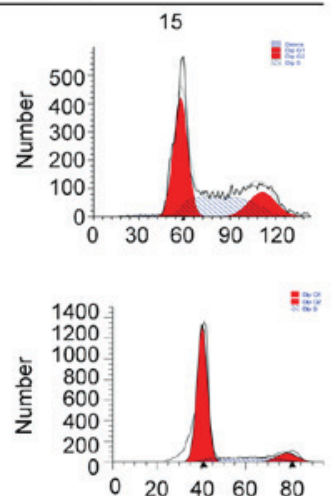
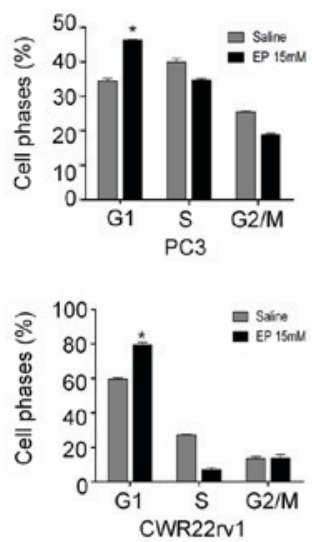

C
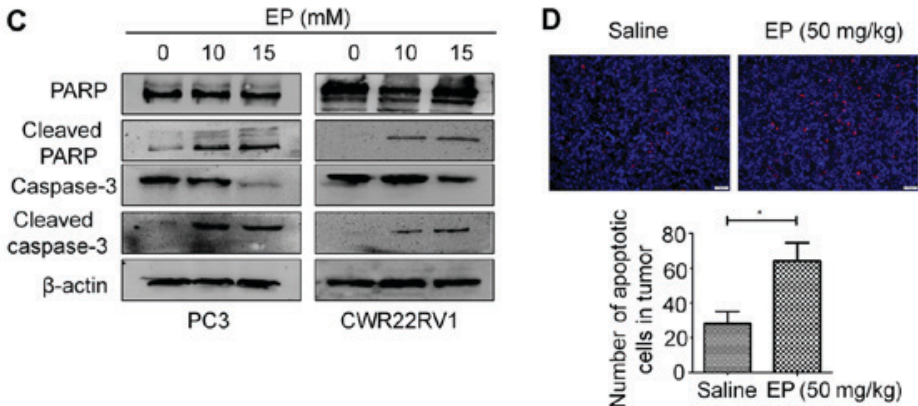

Figure 2. EP causes cell cycle arrest and induces apoptosis. (A) EP causes cell cycle arrest of different PCa cells in G0/G1 phases of the cell cycle. The data represented the mean $\pm \mathrm{SEM}\left({ }^{*} \mathrm{P}<0.05, \mathrm{n}=3\right)$. Comparisons shown: "significant differences between EP treatment groups and Saline vehicle control groups. (B) The expression of P21, CDK4, Cyclin D1 was detected by western blot in vitro. (C) The expression of PARP and caspase-3, cleaved PARP and caspase-3 on protein level was detected by western blot in vitro. (D) TUNNEL staining showed the apoptotic cells in two groups. Magnification, x200 ("P $<0.05$ for Saline vs. Saline + EP $(50 \mathrm{mg} / \mathrm{kg}))$. EP, ethyl pyruvate; PCa, prostate cancer; means \pm SEM, mean \pm standard error of mean.

A

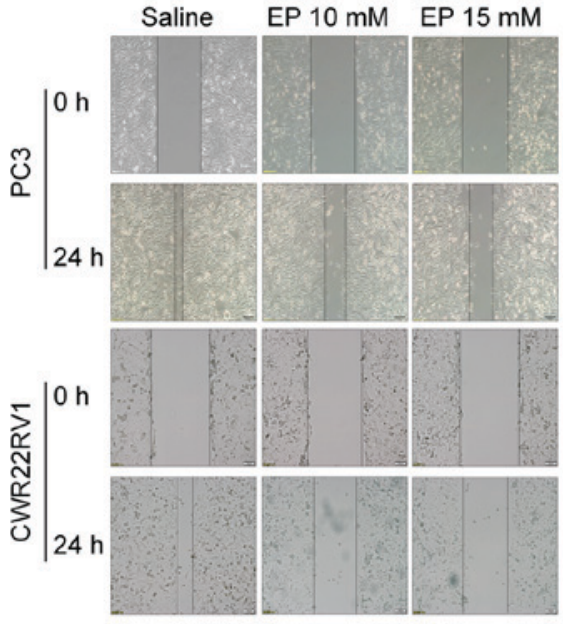

C

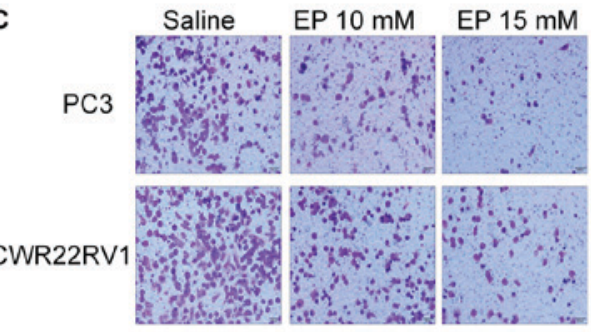

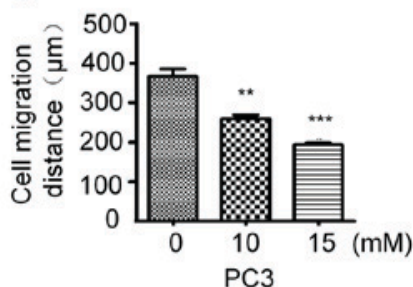
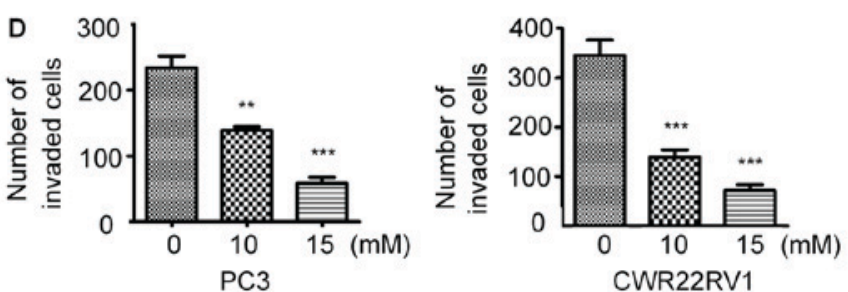

E

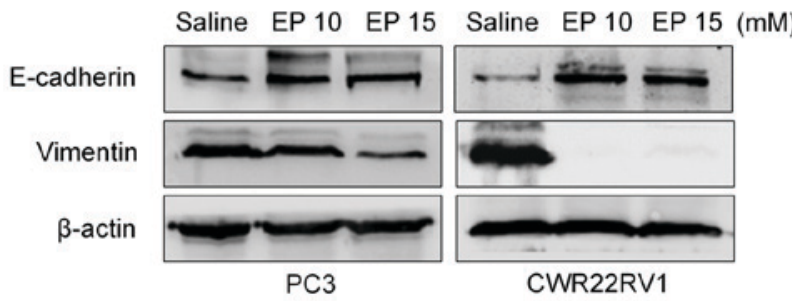

Figure 3. EP inhibited PCa cells invasion and migration. (A) EP reduced migration ability in PCa cells, as revealed by the cell wound healing assay. (B) Quantification. (C) EP Inhibition of cell invasion of CWR22RV1 and PC3 cells after EP treatment for $24 \mathrm{~h}$ (magnification, x200). (D) Dose-dependent inhibition of cell invasion of CWR22RV1 and PC3 cells treated with EP. Data represented means \pm SEM of three independent experiments. (E) Total cell lysates were extracted from CWR22RV1 and PC3 cells after EP treatment, and the expressions of E-cadherin, Vimentin protein were detected by western blot. $\beta$-actin was used as a loading control. The data represented the mean $\pm \mathrm{SEM}\left({ }^{* *} \mathrm{P}<0.01,{ }^{* * * *} \mathrm{P}<0.001, \mathrm{n}=3\right)$. Comparisons shown: "significant differences between EP treatment groups and Saline vehicle control groups. EP, ethyl pyruvate; PCa, prostate cancer; means \pm SEM, mean \pm standard error of mean. 
A
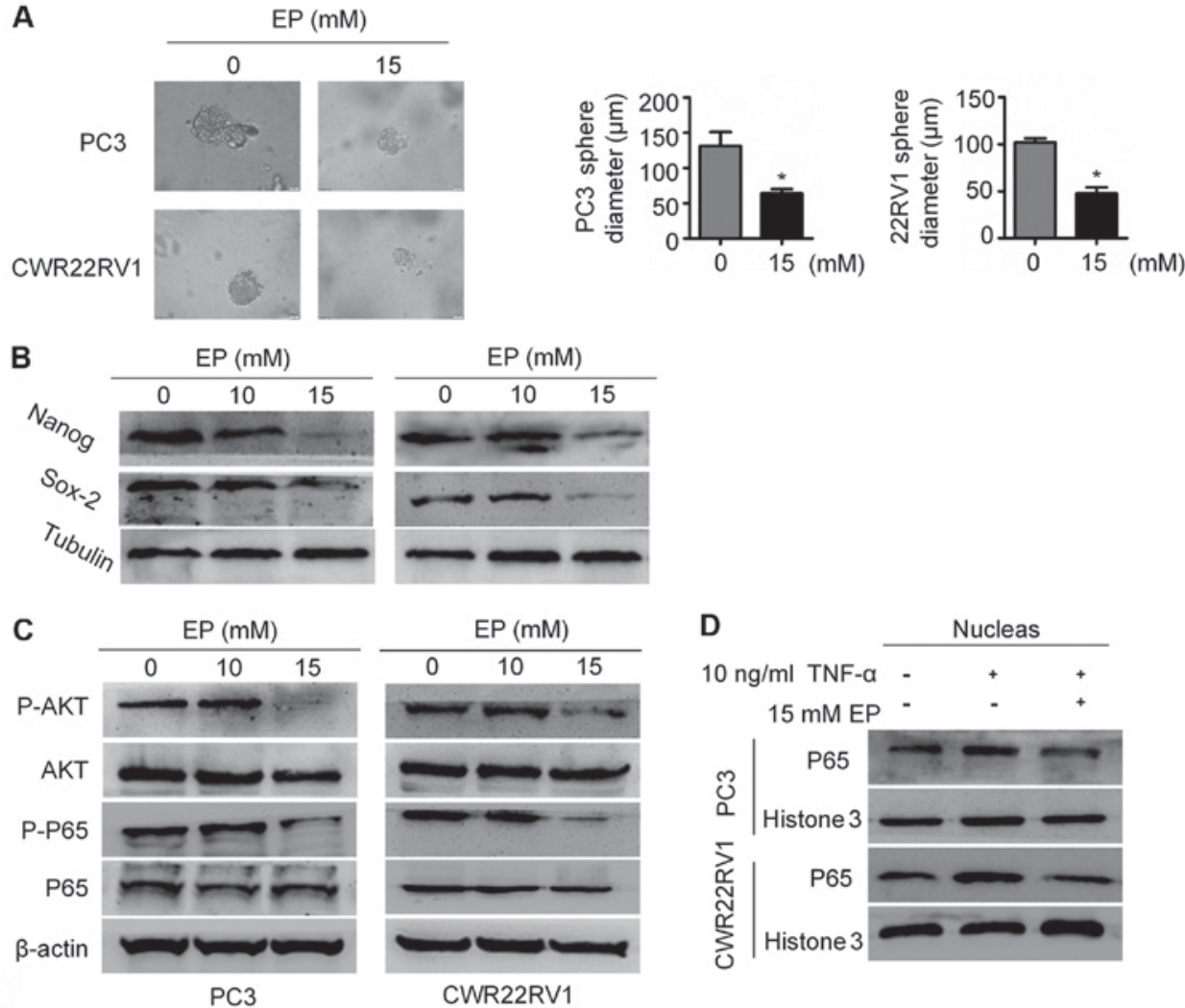

Figure 4. EP suppressed CSC properties and CSC markers expression and AKT/NF- $\mathrm{BB}$ signaling pathway. (A) The representative pictures of tumorspheres of PC3 and CWR22Rv1 cells with or without EP treatment (magnification, x400). Quantification of the suppressive effect of EP on sphere formation of PC3 and CWR22Rv1 cells. The data represented means \pm SEM ("P $<0.05, n=3)$. "Significant differences between EP treatment groups and Saline vehicle control groups. (B) PC3 and CWR22Rv1 cells were treated with EP, and then the level of NANOG and SOX2 were determined by western blotting assay as described in the Materials and methods section. Results (mean \pm SEM) were obtained from three independent experiments. " $\mathrm{P}<0.05$ between indicated groups. (C) PCa cells were treated with EP $(10$ and $15 \mathrm{mM})$ for $48 \mathrm{~h}$. Cell lysates were immunoblotted with specific antibodies to P-AKT, AKT, P65, P-P65. $\beta$-actin was used for normalization. (D) PCa cells were stimulated by $10 \mathrm{ng} / \mathrm{ml}$ TNF- $\alpha$ for $2 \mathrm{~h}$ after treatment with EP $(15 \mathrm{mM})$ for $10 \mathrm{~h}$. The amount of nuclear NF-kB/p65 level was analyzed by fractionation and western blotting assay. Histone 3 was used for normalization. EP, ethyl pyruvate; PCa, prostate cancer; means \pm SEM, mean \pm standard error of mean.

EP has been established to induce apoptosis in many cancer cell lines $(15,18)$. The levels of caspase-3 and PARP were therefore examined in PCa cells by western blotting. The cleaved forms of PARP and caspase-3 were increased in both CWR22RV1 and PC3 cells following treatment with 10 or $15 \mathrm{mM}$ EP for $48 \mathrm{~h}$ (Fig. 2C). In vivo, TUNEL staining also demonstrated an increase in tumor cell apoptosis after treated with EP (P<0.05; Fig. 2D).

EP inhibits the migration and suppresses the metastatic potential of PCa cells. To determine whether EP inhibits PCa cell motility, a wound-healing assay was performed. EP reduced the migratory capacities of both CWR22RV1 and PC3 cells in a concentration-dependent manner (Fig. 3A and B). Furthermore, the results of a Transwell invasion assay revealed that there were fewer invading cells in the treatment group as compared with the control group (Fig. 3C and D).

EMT serves a key role in cancer metastasis and invasion $(21,22)$. The expression of epithelial markers including E-cadherin and mesenchymal markers including vimentin were therefore examined in PC3 and CWR22RV1 cells treated with EP. The level of E-cadherin was upregulated, whereas that of vimentin was downregulated, in the treatment group relative to the control, suggesting that EP prevents metastasis and invasion by blocking EMT (Fig. 3E).

EP suppresses CSC stemness and $A K T / N F-\kappa B$ signaling. Given that EMT is associated with stemness, the effects of EP on CSCs were examined. The results of a sphere formation assay demonstrated that EP suppressed the stem cell characteristics of PC3 and CWR22RV1 cells: The diameter of tumor spheres was smaller in the EP treatment group than in the control group $(\mathrm{P}<0.05$; Fig. $4 \mathrm{~A})$, which was associated with the downregulation of the stemness markers Nanog and (sex determining region Y)-box 2 (Sox2; Fig. 4B).

Aberrant activation of NF- $\mathrm{kB}$ signaling in $\mathrm{PCa}$ has been associated with metastatic progression $(23,24)$. NF- $\mathrm{\kappa B}$ signaling serves an important role in EMT and the maintenance of CSC stemness $(25,26)$. It was observed here that EP treatment reduced p65 phosphorylation (Fig. 4C), which is required for NF- $\mathrm{kB} / \mathrm{p} 65$ nuclear translocation. Accordingly, EP blocked NF- $\mathrm{KB}$ nuclear accumulation induced by TNF- $\alpha$ (Fig. 4D). To clarify the mechanism underlying the inhibition of NF- $\mathrm{KB}$ signaling by EP, AKT expression and phosphorylation was evaluated by western blotting. EP treatment decreased the levels of p-AKT in both PC3 and CWR22RV1 cells (Fig. 4C). These results suggest that EP suppresses EMT in part by targeting the AKT/NF-кB pathway. 


\section{Discussion}

The antitumor effects of EP have been reported; however, they have not been previously investigated in the context of PCa. The results of the present study indicate that EP inhibits the proliferation of PCa cells by inducing cell cycle arrest and apoptosis and suppressing tumor growth in vivo, consistent with previous reports on gallbladder cancer cells and hepatocellular carcinoma $(16,18)$. Additionally, it was observed that EP targets EMT and CSCs through negative regulation of AKT/NF- $\mathrm{BB}$ signaling.

The loss of epithelial phenotypes such as spindle morphology and intercellular adhesion, and the acquisition of mesenchymal characteristics including high migration and invasion capacities and lower cell-extracellular adhesion are two major events that occur when epithelial cells undergo EMT (7). Studies demonstrate that EMT is associated with CRPC $(27,28)$. Sun et al (29) identified that castration may cause EMT, evidenced by decreased expression of epithelial markers (including E-cadherin) and increased protein levels of mesenchymal markers (including N-cadherin, Slug, Zeb1 and Twist1), in both human LuCaP35 prostate cancer xenograft tumors and normal mouse prostate tissue following androgen deprivation, And similar changes have also been identified in human samples undergoing ADT (29). EMT is driven by EMT-inducing transcription factors (including Snail, Slug, Zeb1, Zeb2 and Twist), some of which have been reported to participate in the development of CRPC (7). Zeb1 expression in CRPC is higher than in androgen-sensitive prostate cancer and may be a reliable indicator of poor prognosis in patients (30). In addition, stable overexpression of Twist in a prostate cancer cell line served a fundamental role in the formation and progression of CRPC by mediating EMT and CSCs (27). Shiota et al (31) reported that castration-induced oxidative stress may promote androgen receptor (AR) overexpression through Twistl overexpression, which could result in a gain of castration resistance. Furthermore, facilitation of castration resistance by Slug in prostate cancer has been reported by Wu et al (32). Slug, another transcription factor driver of EMT, can not only augment the expression of AR but also enhance AR transcriptional activities with or without androgen as a novel coactivator for AR (32). Self-renewal and differentiation into diverse tumor cells are unique characteristics of CSCs, which have been hypothesized to be a subpopulation of tumor cells that drive treatment resistance in prostate cancer due to growth independence from androgen stimulation (33). CSC biomarkers, including Nanog, Sox2, Nkx3.1, PSA-/LO and Bmi-1, have been applied to identify and isolate CSCs in solid tumors including prostate cancer (34). A number of studies have observed that these biomarkers associated with CSCs may serve a central role in the progression of CRPC (35). Overexpression of Nanog facilitates the self-renewal and tumor sphere-formation ability of prostate cancer cells and promotes castration-resistant tumor growth in an androgen-deprived environment $(36,37)$. Furthermore, Rybak et al (38) demonstrated that Sox 2 is critically associated with the self-renewal and tumor progression of human prostate cancer. In addition, Sox 2 has also been demonstrated as a factor associated with castration-resistant tumor growth and to be negatively regulated by the AR signaling axis (39).
Overall, these aforementioned studies suggest that both EMT and CSCs are responsible for progression and treatment resistance in prostate cancer. Accordingly, treatment capable of reversing EMT phenotypes or suppressing CSCs may become a viable alternative in the future for CRPC therapy. In the current study, it was indicated that EP effectively reversed the EMT phenotype in the CRPC cell lines 22RV1 and PC3, as evidenced by decreased expression of vimentin and an increased protein level of E-cadherin. Furthermore, it was demonstrated that the ability of tumor-sphere formation in CRPC cells was suppressed following EP treatment. Notably, the biomarkers of CSCs, including Nanog and Sox 2, decreased in CRPC cells following EP treatment, which indicates that EP may suppress the CSC subpopulation in prostate cancer. These results indicate that EP may be an alternative therapy for future CRPC treatment by targeting the EMT phenotype and CSCs.

The NF- $\kappa$ B family, an important class of transcriptional regulators, comprises five members including RelA (p65),

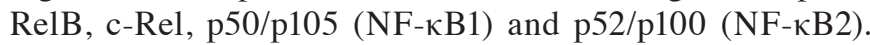
$\mathrm{NF}-\kappa \mathrm{B}$ binds to inhibitor $\kappa \mathrm{B}(\mathrm{I} \kappa \mathrm{B})$ protein in the cytoplasm in an inactive state. In a pathological state, the IкB kinase (IKK) complex is activated and subsequently induces the phosphory-

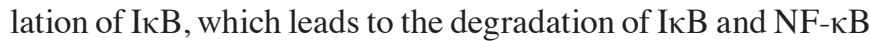
translocation to nucleus (40). Increasing results indicate that the NF- $\mathrm{kB}$ transcription factor family is a crucial mediator of EMT and CSCs $(25,26)$. Certain studies have demonstrated that NF-kB may bind to promoters of genes associated with EMT and CSCs, including Snail, Slug and Twist, to increase transcription $(25,41)$. Ozes et al (42) reported that AKT was involved in the activation of NF- $\kappa B$ by mediating the phosphorylation of IKKA which is responsible for the activation of its downstream target IкB. Certain reports indicate that EP inhibits the phosphorylation of AKT (18) and suppresses the DNA-binding activity of NF- $\mathrm{KB}$ via direct modification of $\mathrm{p} 65$ at Cys 38 (43). In the current study, it was observed that EP could inhibit the phosphorylation of both AKT and p65; in addition, nuclear translocation of p65 induced by TNF- $\alpha$ was blocked by EP. These results indicate that EP suppresses EMT and CSCs by negatively regulating the AKT/NF- $\mathrm{KB}$ pathway.

Furthermore, the AKT/NF- $\kappa \mathrm{B}$ signaling pathway might drive the progression of CRPC by another mechanism besides the induction of EMT or CSCs. Activation of NF- $\mathrm{KB}$ mediated by PI3K/AKT increases the expression of AR via NF- $\mathrm{KB}$ binding to the AR promoter (44). CRPC, previously defined as hormone-refractory prostate cancer, remains to be considered as androgen-dependent (45), which indicates that targeting AR remains an effective therapeutic strategy for CRPC. The present data demonstrated that EP suppressed the phosphorylation of AKT and p65; however, whether the effect of EP on AR signaling axis via regulation of the AKT/NF- $\kappa B$ pathway is unclear and worthy of further examination.

A phase II multicenter double-blind placebo-controlled study has demonstrated that EP administration in patients undergoing higher-risk cardiac surgery has no side effects (46). In addition, another study indicated that EP exerted a potent effect on leukemia cells, while being safe for normal blood cells (14). Therefore, taken together the available data on EP suggest that it may be an effective and safe therapeutic agent for the treatment of prostate cancer. 


\section{Acknowledgements}

Not applicable.

\section{Funding}

This work was supported by the Science and Technology Planning Projects of Guangdong Province (grant nos. 2013B051000050, 2014A020212538, 2014A020212663 and 2016A020215175); Natural Science Foundation of Guangdong Province (grant nos. 2016A030313583 and 508312335027); Medical Scientific Research Foundation of Guangdong Province (grant no. A2016555); Science and Technology Planning Project of Guangzhou (grant no. 201704020070); and Outstanding Youths Development Scheme of Nanfang Hospital, Southern Medical University (grant no. 2015J005); National undergraduate innovation training program funded projects of Southern Medical University (grant no. 201612121005).

\section{Availability of data and materials}

The datasets used and/or analyzed during the current study are available from the corresponding author on reasonable request.

\section{Authors' contributions}

This study was conceived and supervised by SCZ and HQ. Experiments were carried out by $\mathrm{BH}$ and DJL. Data analysis was conducted by CW and FPS. XLS provided technical support for western blotting. ZCG, YZY, TX, JJX, SL and YML assisted with experiments.

\section{Ethics approval and consent to participate}

Procedures involving animals were approved by the Institutional Animal Care and Use Committee of Southern Medical University.

\section{Pantient consent for publication}

Not applicable.

\section{Competing interests}

All authors have declared that they have no competing interests.

\section{References}

1. Siegel RL, Miller KD and Jemal A: Cancer statistics, 2018. CA Cancer J Clin 68: 7-30, 2018.

2. Shafi AA, Yen AE and Weigel NL: Androgen receptors in hormone-dependent and castration-resistant prostate cancer Pharmacol Ther 140: 223-238, 2013.

3. Loriot Y, Bianchini D, Ileana E, Sandhu S, Patrikidou A, Pezaro C, Albiges L, Attard G, Fizazi K, De Bono JS and Massard C: Antitumour activity of abiraterone acetate against metastatic castration-resistant prostate cancer progressing after docetaxel and enzalutamide (MDV3100). Ann Oncol 24: 1807-1812, 2013.

4. Schrader AJ, Boegemann M, Ohlmann CH, Schnoeller TJ, Krabbe LM, Hajili T, Jentzmik F, Stoeckle M, Schrader M, Herrmann E and Cronauer MV: Enzalutamide in castration-resistant prostate cancer patients progressing after docetaxel and abiraterone. Eur Urol 65: 30-36, 2014.
5. Chaffer CL, San Juan BP, Lim E and Weinberg RA: Emt, cell plasticity and metastasis. Cancer Metastasis Rev 35: 645-654, 2016.

6. Wang D, Plukker J and Coppes RP: Cancer stem cells with increased metastatic potential as a therapeutic target for esophageal cancer. Semin Cancer Biol 44: 60-66, 2017.

7. Lamouille S, Xu J and Derynck R: Molecular mechanisms of epithelial-mesenchymal transition. Nat Rev Mol Cell Biol 15: 178-196, 2014.

8. Jaworska D, Król W and Szliszka E: Prostate cancer stem cells: Research advances. Int J Mol Sci 16: 27433-27449, 2015.

9. Bitting RL, Schaeffer D, Somarelli JA, Garcia-Blanco MA and Armstrong AJ: The role of epithelial plasticity in prostate cancer dissemination and treatment resistance. Cancer Metastasis Rev 33: 441-468, 2014.

10. Chen X, Li Q, Liu X, Liu C, Liu R, Rycaj K, Zhang D, Liu B, Jeter C, Calhoun-Davis T, et al: Defining a population of stem-like human prostate cancer cells that can generate and propagate castration-resistant prostate cancer. Clin Cancer Res 22: 4505-4516, 2016.

11. Chen W, Lian J, Ye JJ, Mo QF, Qin J, Hong GL, Chen LW, Zhi SC, Zhao GJ and Lu ZQ: Ethyl pyruvate reverses development of Pseudomonas aeruginosa pneumonia during sepsis-induced immunosuppression. Int Immunopharmacol 52: 61-69, 2017.

12. Fink MP: Ethyl pyruvate: A novel anti-inflammatory agent. J Intern Med 261: 349-362, 2007.

13. Pellegrini L, Xue J, Larson D, Pastorino S, Jube S, Forest KH, Saad-Jube ZS, Napolitano A, Pagano I, Negi VS, et al: HMGB1 targeting by ethyl pyruvate suppresses malignant phenotype of human mesothelioma. Oncotarget 8: 22649-22661, 2017.

14. Birkenmeier G, Hemdan NY, Kurz S, Bigl M, Pieroh P, Debebe T, Buchold M, Thieme R, Wichmann G and Dehghani F: Ethyl pyruvate combats human leukemia cells but spares normal blood cells. PLoS One 11: e0161571, 2016.

15. Liang X, Chavez AR, Schapiro NE, Loughran P, Thorne SH, Amoscato AA, Zeh HJ, Beer-Stolz D, Lotze MT and de Vera ME: Ethyl pyruvate administration inhibits hepatic tumor growth. J Leukoc Biol 86: 599-607, 2009.

16. Li ML, Wang XF, Tan ZJ, Dong P, Gu J, Lu JH, Wu XS, Zhang L, Ding QC, Wu WG, et al: Ethyl pyruvate administration suppresses growth and invasion of gallbladder cancer cells via downregulation of HMGB1-RAGE axis. Int J Immunopathol Pharmacol 25: 955-965, 2012.

17. Park SY, Yi EY, Jung M, Lee YM and Kim YJ: Ethyl pyruvate, an anti-inflammatory agent, inhibits tumor angiogenesis through inhibition of the NF- $\mathrm{KB}$ signaling pathway. Cancer Lett 303: 150-154, 2011.

18. Cheng P, Dai W, Wang F, Lu J, Shen M, Chen K, Li J, Zhang Y, Wang C, Yang J, et al: Ethyl pyruvate inhibits proliferation and induces apoptosis of hepatocellular carcinoma via regulation of the HMGB1-RAGE and AKT pathways. Biochem Biophys Res Commun 443: 1162-1168, 2014.

19. Lv D, Wu H, Xing R, Shu F, Lei B, Lei C, Zhou X, Wan B, Yang Y, Zhong L, et al: HnRNP-L mediates bladder cancer progression by inhibiting apoptotic signaling and enhancing MAPK signaling pathways. Oncotarget 8: 13586-13599, 2017.

20. Zhong D, Zhang HJ, Jiang YD, Wu P, Qi H, Cai C, Zheng SB and Dang Q: Saikosaponin-d: A potential chemotherapeutics in castration resistant prostate cancer by suppressing cancer metastases and cancer stem cell phenotypes. Biochem Biophys Res Commun 474: 722-729, 2016.

21. Ye X and Weinberg RA: Epithelial-mesenchymal plasticity: A central regulator of cancer progression. Trends Cell Biol 25: 675-686, 2015

22. Karlsson MC, Gonzalez SF, Welin J and Fuxe J: Epithelial-mesenchymal transition in cancer metastasis through the lymphatic system. Mol Oncol 11: 781-791, 2017.

23. Lessard L, Karakiewicz PI, Bellon-Gagnon P, Alam-Fahmy M, Ismail HA, Mes-Masson AM and Saad F: Nuclear localization of nuclear factor-kappaB p65 in primary prostate tumors is highly predictive of pelvic lymph node metastases. Clin Cancer Res 12: 5741-5745, 2006.

24. Ismail HA, Lessard L, Mes-Masson AM and Saad F: Expression of NF-kappaB in prostate cancer lymph node metastases. Prostate 58: 308-313, 2004.

25. Min C, Eddy SF, Sherr DH and Sonenshein GE: NF-kappaB and epithelial to mesenchymal transition of cancer. J Cell Biochem 104: 733-744, 2008.

26. Rajasekhar VK, Studer L, Gerald W, Socci ND and Scher HI: Tumour-initiating stem-like cells in human prostate cancer exhibit increased NF-kB signalling. Nat Commun 2: 162, 2011. 
27. NakazawaMandKyprianouN:Epithelial-mesenchymal-transition regulators in prostate cancer: Androgens and beyond. J Steroid Biochem Mol Biol 166: 84-90, 2017.

28. Li P, Yang R and Gao WQ: Contributions of epithelial-mesenchymal transition and cancer stem cells to the development of castration resistance of prostate cancer. Mol Cancer 13: 55, 2014.

29. Sun Y, Wang BE, Leong KG, Yue P, Li L, Jhunjhunwala S, Chen D, Seo K, Modrusan Z, Gao WQ, et al: Androgen deprivation causes epithelial-mesenchymal transition in the prostate: Implications for androgen-deprivation therapy. Cancer Res 72: 527-536, 2012.

30. Figiel S, Vasseur C, Bruyere F, Rozet F, Maheo K and Fromont G Clinical significance of epithelial-mesenchymal transition markers in prostate cancer. Hum Pathol 61: 26-32, 2017.

31. Shiota M, Yokomizo A, Tada Y, Inokuchi J, Kashiwagi E, Masubuchi D, Eto M, Uchiumi T and Naito S: Castration resistance of prostate cancer cells caused by castration-induced oxidative stress through Twist 1 and androgen receptor overexpression. Oncogene 29: 237-250, 2010.

32. Wu K, Gore C, Yang L, Fazli L, Gleave M, Pong RC, Xiao G, Zhang L, Yun EJ, Tseng SF, et al: Slug, a unique androgen-regulated transcription factor, coordinates androgen receptor to facilitate castration resistance in prostate cancer. Mol Endocrinol 26: 1496-1507, 2012.

33. Ni J, Cozzi P, Hao J, Duan W, Graham P, Kearsley J and Li Y: Cancer stem cells in prostate cancer chemoresistance. Curr Cancer Drug Targets 14: 225-240, 2014.

34. Yun EJ, Zhou J, Lin CJ, Hernandez E, Fazli L, Gleave M and Hsieh JT: Targeting cancer stem cells in castration-resistant prostate cancer. Clin Cancer Res 22: 670-679, 2016.

35. Wang X, Kruithof-de Julio M, Economides KD, Walker D, Yu H, Halili MV, Hu YP, Price SM, Abate-Shen C and Shen MM: A luminal epithelial stem cell that is a cell of origin for prostate cancer. Nature 461: 495-500, 2009.

36. Jeter CR, Liu B, Liu X, Chen X, Liu C, Calhoun-Davis T, Repass J, Zaehres H, Shen JJ and Tang DG: NANOG promotes cancer stem cell characteristics and prostate cancer resistance to androgen deprivation. Oncogene 30: 3833-3845, 2011.

37. Jeter CR, Badeaux M, Choy G, Chandra D, Patrawala L, Liu C, Calhoun-Davis T, Zaehres H, Daley GQ and Tang DG: Functional evidence that the self-renewal gene NANOG regulates human tumor development. Stem Cells 27: 993-1005, 2009.
38. Rybak AP and Tang D: SOX2 plays a critical role in EGFR-mediated self-renewal of human prostate cancer stem-like cells. Cell Signal 25: 2734-2742, 2013.

39. Kregel S, Kiriluk KJ, Rosen AM, Cai Y, Reyes EE, Otto KB, Tom W, Paner GP, Szmulewitz RZ and Vander Griend DJ: Sox 2 is an androgen receptor-repressed gene that promotes castration-resistant prostate cancer. PLoS One 8: e53701, 2013.

40. Taniguchi $\mathrm{K}$ and Karin M: NF- $\kappa \mathrm{B}$, inflammation, immunity and cancer: Coming of age. Nat Rev Immunol 18: 309-324, 2018.

41. Kotiyal S and Bhattacharya S: Breast cancer stem cells, emt and therapeutic targets. Biochem Biophys Res Commun 453: 112-116, 2014.

42. Ozes ON, Mayo LD, Gustin JA, Pfeffer SR, Pfeffer LM and Donner DB: NF-kappaB activation by tumour necrosis factor requires the Akt serine-threonine kinase. Nature 401: 82-85, 1999.

43. Han Y, Englert JA, Yang R, Delude RL and Fink MP: Ethyl pyruvate inhibits nuclear factor-kappaB-dependent signaling by directly targeting p65. J Pharmacol Exp Ther 312: 1097-1105, 2005.

44. Lee SO, Lou W, Nadiminty N, Lin X and Gao AC: Requirement for NF-(kappa)B in interleukin-4-induced androgen receptor activation in prostate cancer cells. Prostate 64: 160-167, 2005.

45. Penning TM: Mechanisms of drug resistance that target the androgen axis in castration resistant prostate cancer (CRPC). J Steroid Biochem Mol Biol 153: 105-113, 2015.

46. Bennett-Guerrero E, Swaminathan M, Grigore AM, Roach GW, Aberle LG, Johnston JM and Fink MP: A phase II multicenter double-blind placebo-controlled study of ethyl pyruvate in high-risk patients undergoing cardiac surgery with cardiopulmonary bypass. J Cardiothorac Vasc Anesth 23 324-329, 2009.

This work is licensed under a Creative Commons Attribution-NonCommercial-NoDerivatives 4.0 International (CC BY-NC-ND 4.0) License. 